\title{
Reseña del libro Motivación y personalidad de Abraham Maslow (1991)*
}

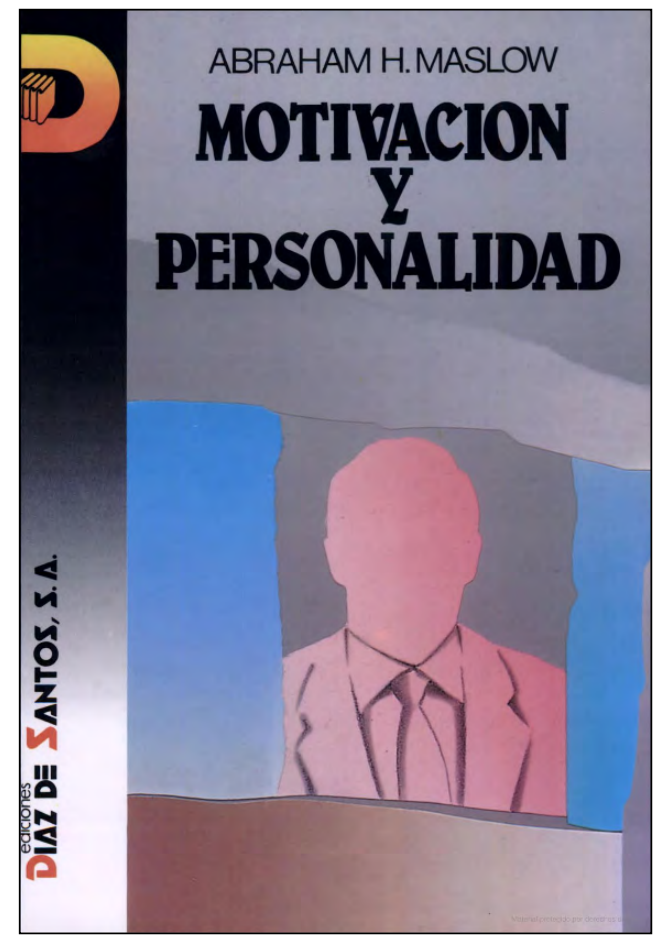

Portada del libro Motivación y personalidad

Fuente: Maslow (1991).

Yair Cafiel Cuello

Universidad Metropolitana de Educación, Ciencia y Tecnología, Umecit,

Ciudad de Panamá, Panamá

yaircafiel@unicesar.edu.co

https://orcid.org/0000-0002-6685-6907

Cómo citar: Cafiel Cuello, Y. (2021). Reseña del libro Motivación y personalidad de Abraham Maslow (1991). Ciencias Sociales y Educación, 10(20), 327-333. https://doi.org/10.22395/csye.v10n20a16

Recibido: 17 de julio de 2021

Aprobado: 28 de julio de 2021 
En el libro titulado Motivación y personalidad de Abraham Maslow, el autor expone la temática en cuatro partes. La primera parte, llamada "Teoría de la motivación," se encuentra constituida por capítulos; uno de ellos es "La jerarquía de las necesidades". En este aparte se aborda lo correspondiente a las diferencias entre las necesidades superiores y las inferiores, y las consecuencias de la jerarquía de las necesidades.

En este orden de ideas, el capítulo "La jerarquía de las necesidades" se encuentra clasificada en necesidades superiores e inferiores, con propiedades completamente desiguales, pero forman parte de la naturaleza humana. En ese sentido, se resalta el animal biológico y sus necesidades instintivas propias de cualquier ser vivo de la naturaleza, como la alimentación, la reproducción, la protección y otras necesidades intrínsecas o extrínsecas convenientes de la especie humana. Todas hacen parte del desarrollo y la cultura de cada individuo o sociedad como el amor, la belleza, la estima, la autorrealización, entre otras.

Las necesidades superiores y las inferiores presentan una marcada diferencia, de tal manera que unas serán más fuertes que otras al momento de satisfacerlas, por lo que potencialmente el ser humano sentirá siempre la necesidad de satisfacer inicialmente unas necesidades básicas y, posteriormente, otras de mayor jerarquía, pero de menos importancia biológica. Por su parte, Maslow (1991) dice que

las necesidades fisiológicas son más fuertes que las de seguridad, que son más fuertes que las necesidades de amor, que a su vez son más fuertes que las necesidades de estima, que son más fuertes que aquellas necesidades de autorrealización. (p. 88)

Por otra parte, el autor enfoca las consecuencias de la jerarquía de las necesidades en tratar de reconocer si las necesidades superiores, tales como la amistad, la intimidad, el respeto, el éxito, la autorrealización, entre otras, son tan instintivas o biológicas como las necesidades inferiores (alimentación, respiración, reproducción, seguridad y salud, entre otras), de las cuales pueden salir diversas repercusiones, pero se limita a explicar unas cuantas.

Al respecto, Maslow (1991) manifiesta, en sus repercusiones, que dentro de la naturaleza del ser humano hay un animal muy especial, dado que nuestras necesidades no son totalmente ciegas, sino que dependen y se modifican según la cultura, la realidad y la posibilidad que tenga cada individuo. Así, le da una gran importancia a las acciones cognitivas y voluntarias o instintivas de la naturaleza animal. Estas últimas no son tan instintivas como en el resto de los animales de la naturaleza. Estas diferencias instintivas que se hacen fuertes, indeseables e inmutables en el común de los animales de la naturaleza, contradictoriamente, se hacen débiles en las necesidades básicas del ser 
humano -aunque también instintivas-, dado que la conciencia que nos impulsa a saber realmente lo que queremos y necesitamos como proyecto de vida se convierte en un logro psicológico difícil para satisfacer. Asimismo, cuanto más superiores sean las necesidades, estas serán más débiles y más fáciles de cambiar y suprimir en el desarrollo del individuo.

Es de aclarar que las necesidades superiores no son clasificadas como malas, sino que simplemente se ubican como neutrales o buenas. No obstante, dentro del instinto humano, lo que queda es tan débil que permanentemente necesita de la protección contra la cultura, la educación, el aprendizaje, lo que finalmente se traduce, en una palabra, contra la opresión del entorno. Lo superior siempre se desarrollará sobre la base de lo inferior, y en la medida en que quede bien estructurado, podría llegar a ser relativamente independiente de lo inferior.

Finalmente, según la teoría de la supervivencia de Charles Darwin, todos los seres vivos lucharán por mantenerse con vida, donde los más débiles desaparecerán. En la especie humana, no solo es bueno sobrevivir, sino que también se busca ese complemento que caracteriza y diferencia a la humanidad como la potencialidad de tener felicidad, serenidad, experiencias y transcendencia, entre otras.

\section{Pregunta orientadora}

¿En qué consisten las necesidades inferiores y superiores que hacen parte del desarrollo y del proyecto de vida de los seres humanos?

\section{El objetivo del texto}

Caracterizar las necesidades inferiores y superiores que hacen parte del desarrollo y del proyecto de vida de los seres humanos.

\section{Tesis principal del texto}

Hay necesidades básicas esenciales y mínimas que todos los seres vivos requieren para sobrevivir, incluyendo a los seres humanos.

La satisfacción en los seres humanos de las diferentes necesidades, tanto inferiores como superiores, contribuye de una u otra manera en el proceso de formación y desarrollo de cada individuo. 


\section{Argumentación del texto a favor de la tesis}

En general, para sobrevivir, todos los seres vivos de la naturaleza necesitan de unas condiciones mínimas de desarrollo para garantizar la supervivencia de sí mismos y la permanencia de la especie. Estas condiciones se cumplen cuando el individuo puede mantener su homeóstasis. Para ello, necesita, inicialmente, satisfacer fisiológicamente al cuerpo de oxígeno, alimentos, agua y demás nutrientes esenciales que contribuyen para que, al menos, el ser se mantenga con vida.

Al satisfacer las necesidades básicas fisiológicas, el ser humano, a diferencia del resto de los seres vivos, ha desarrollado otros estados de necesidades consideradas motivacionales, presentes y desarrolladas únicamente en la especie humana. Dichas necesidades motivacionales llevan a la persona a desear o satisfacer objetos o momentos que no se presentan en ninguna otra especie de la naturaleza, como obtener una prenda de vestir, tener compañía, un vehículo de transporte, amistad, recreación, prestigio, entre otras.

En su efecto, el ser humano comienza a satisfacer, desde su nacimiento, sus necesidades. Por ende, las primeras en ser satisfechas serían las de supervivencia o fisiológicas, y posteriormente - y de forma simultánea- se comenzarían a generar y satisfacer otras necesidades según el caso y las condiciones. Al respecto, Maslow (1991) afirma que

el humano es un animal necesitado y raramente alcanza un estado de completa satisfacción, excepto en breves períodos de tiempo. Tan pronto se ha satisfecho un deseo, aparece otro. Cuando éste [sic] se satisface, otro nuevo se sitúa en primer plano. (p. 9)

Por su parte, Carver y Scheier (1997) hacen una reflexión sobre la relación entre conducta humana y las necesidades básicas, donde una es el reflejo de la otra. La necesidad es un estado interno no satisfactorio: la falta de algo que se requiere para el bienestar. Estos estudios fueron iniciados por Henry Murray (1938), citado por Carver y Scheier (1997), quien definió la necesidad como una fuerza directriz interna que determina la forma en que la gente decide responder a los objetos o a las situaciones de su entorno.

Es claro, entonces, que hace parte de la especie humana, independientemente cual sea su origen, raza, posición geográfica, estrato socioeconómico y demás, estar deseando algo de forma permanente durante su existencia. Estas necesidades, según su jerarquía, pueden ser satisfechas o no, lo cual depende de sus condiciones familiares, sociales, económicas, motivacionales, políticas, entre otras. Asimismo, el ser humano experimenta y goza de un alto grado de satisfacción emocional cuando finalmente logra obtener una o más necesi- 
dades que fueron, en su momento, deseadas, ya sea por impulsos netamente fisiológicos del cuerpo o por efecto de un locus de causalidad motivacional intrínseco o extrínseco.

Por consiguiente, Abraham Maslow (1970), citado por Arends (2007), reverenciado como uno de los más importantes psicólogos estadounidenses de mediados del siglo veinte, planteó que los seres humanos tienen una jerarquía de necesidad que se esfuerzan por satisfacer. Maslow (1991), categorizó estas necesidades en cinco niveles: las inferiores, donde existen necesidades de satisfacer los requisitos fisiológicos básicos, la seguridad y la afiliación; y las superiores, que son más complejas y se refieren a las necesidades humanas de crecimiento tales como el reconocimiento y la autorrealización. Asimismo, explica que, potencialmente y de forma relativa, las personas se esfuerzan por satisfacer las necesidades de orden superior. Cuando esto se presenta, se han satisfecho las necesidades físicas y las necesidades de amor y autoestima. De esta manera, jerarquiza la pirámide de necesidades, donde las de nivel superior se satisfacen solo si las del nivel inferior se satisficieron.

Cabe señalar que, subjetivamente, puede existir de forma relativa un orden de elección o preferencia al momento de satisfacer alguna necesidad. Pero ese orden, dentro de las estimaciones generales, siempre se desarrollará desde lo inferior a lo superior, donde la satisfacción de las necesidades superiores dependerá directamente de la satisfacción de las necesidades inferiores. Al respecto, Maslow (1991) dice:

La necesidad superior está en el nivel más alto del desarrollo evolutivo. Compartimos la necesidad de alimento con todo lo vivo, necesidad de amor con (quizá) los simios, necesidad de autorrealización con nadie. Cuanto más superior la necesidad, es más específicamente humana. (p. 88)

Consecuentemente, Maslow (1991), expresa que las necesidades superiores se llevan a cabo de forma posterior a la ontogenia. Esto quiere decir que, al nacer, el individuo comienza por manifestar sus necesidades físicas y probablemente la correspondiente a la seguridad. En la medida que va desarrollando y creciendo en sus primeros meses, comienza a mostrar la satisfacción de otras necesidades como afecto y cariño, y en ese orden de desarrollo se continuarán manifestando diversas necesidades que a su vez serán satisfechas según las condiciones dadas. Cuando el individuo se encuentra en el estatus de satisfacción de necesidades muy superiores, estas serán menos imperiosas para la supervivencia, es decir, será más fácil dejar de satisfacerlas y prescindir sin comprometer la existencia misma.

En este mismo orden de ideas, cuando la persona se encuentra satisfaciendo las necesidades del orden superior, trae como significado que goza de una alta 
eficiencia biológica, tiene una mayor supervivencia, así como disfruta de escasas enfermedades, felicidad profunda, buen sueño, buena dieta, más serenidad, riqueza en la vida interior, entre otras. Es de aclarar que cuando se satisfacen las necesidades superiores a este nivel, son subjetivas según cada individuo y menos urgentes de ser satisfechas. Por lo tanto, la búsqueda de la satisfacción de las necesidades superiores representa una tendencia general hacia el goce de la tranquilidad y una buena salud. En pocas palabras, se encuentran más cerca de la autorrealización.

Por consiguiente, las necesidades del orden superior requieren de muchas condiciones previas para poderlas satisfacer, de tal manera que, para gozar de alguna de estas necesidades, primeramente se requiere de la satisfacción de necesidades del orden inferior. Tal es el caso donde la necesidad de seguridad demanda menor cantidad de satisfacciones que la necesidad de amor. Esto conlleva a deducir que el desarrollo y el cumplimiento de un buen proyecto de vida es más complejo al intentar satisfacer necesidades del nivel superior, para las cuales se requiere de un mayor tiempo para satisfacerlas, medios y metas parciales, fases preparatorias y subordinadas.

Asimismo, para que las necesidades del nivel superior sean satisfechas, también requieren de unas mejores condiciones externas, las cuales están relacionadas con el ambiente familiar, la cultura, los aspectos económicos, la política local, regional y nacional, los procesos educativos, entre otros. Por ende, las personas que han gozado de los dos niveles de necesidades, tanto del superior como del inferior, terminan dándole mayor relevancia a las necesidades del orden superior y, a su vez, están mejor prevenidas para tolerar la carencia parcial de alguna de las necesidades del orden inferior.

Finalmente, con todo lo expuesto en dicho análisis, hay que considerar que las necesidades del nivel inferior son mucho más localizadas, perceptibles y limitadas en comparación con las del nivel superior. Por ejemplo, necesidades localizadas de tipo fisiológicas — como el hambre o la sed- son mucho más corporales que el amor, donde a su vez este lo es más que el respeto y así sucesivamente. Asimismo, haber satisfecho necesidades inferiores es mucho más apreciable y notorio que cuando se satisface una del nivel superior. Y se limitan porque se requiere de una cantidad precisa para satisfacer dicha necesidad, como lo es aplacar o saciar la sensación del hambre con una cantidad definida de alimento, mientras que para otras necesidades como el amor y el respeto no hay límite de satisfacción. 


\section{Conclusión personal que suscita el texto}

Fue muy grato para mí el análisis minucioso de esta temática, por lo que logré entender y profundizar diversos aspectos relacionados con la jerarquía de las necesidades motivacionales en los seres humanos y precisar en detalle las necesidades que corresponden al nivel inferior y superior. Asimismo, me quedó totalmente claro que está comprobado científicamente que el ser humano no actúa por instintos y que detrás de cada acción se encuentra un locus de causalidad motivacional que lo impulsa a pretender y satisfacer las diferentes necesidades que se originan por motivos intrínsecos o extrínsecos.

De la misma manera, este trabajo me llevó a comprender que no hay ninguna duda de que las necesidades humanas se satisfacen en un orden jerárquico, donde primero se tienen que satisfacer las necesidades inferiores, luego las que están relacionadas con los impulsos fisiológicos para que, posteriormente, se puedan satisfacer las del siguiente nivel jerárquico, y así sucesivamente. Esta temática es fundamental y precisa para abordar la tesis principal de la investigación doctoral que se encuentra en curso, titulada "Modelo teórico basado en la jerarquía de las necesidades de Maslow y su influencia en el aprendizaje del área de ciencias naturales en los estudiantes de educación básica secundaria de las instituciones educativas del núcleo 3 de la ciudad de Valledupar-Cesar", así como para ir contextualizando las realidades de la población estudiantil que tengo a cargo en mi hermosa labor como docente.

\section{Referencias}

Arends, R. (2007). Aprender a Enseñar. McGraw-Hill.

Carver, C. y Scheier, M. (1997). Teoría de la Personalidad. Editorial Prentice Hall Hispanoamericana S. A. Maslow, A. (1991). Motivación y personalidad. Ediciones Díaz de Santos. 